\title{
Drift Wave Simulations with Reduced Stellarator Equilibria
}

\author{
J. L.V. Lewandowski \\ Princeton Plasma Physics Laboratory \\ Princeton University \\ P.O. Box 451, Princeton NJ 08543
}

(December 3, 1999)

\begin{abstract}
A three-field model to study drift-resistive, low-frequency waves in low- $\beta$, non-axisymmetric plasmas [J.L.V. Lewandowski, Phys. Plasmas, 4(11) 4023 (1997)] is used to analyze the effect of the inhomogeneities in the stellarator magnetic field on the fastest (linear) growth rate, $\gamma$. Extensive numerical calculations for a toroidal heliac show that not all Fourier components in the representation of the equilibrium configuration are important as far as $\gamma$ is concerned.
\end{abstract}

Pacs \# : 52.35Kt, 52.30Jb, 52.35Ra 
The presence of slow, short-scale instabilities, often called microinstabilities, in magnetically-confined plasmas is a major concern because of the large ('anomalous') perpendicular (cross-field) transport. Substantial efforts have been made to understand microinstability dynamics and the associated transport in tokamak geometry (See, for example, Ref [1]). However very little work has been done on microinstabilities in stellarator geometry.

In the dense, cold edge plasmas of tokamaks and stellarators, one can use a fluid model ${ }^{2}$ to study drift waves in the electrostatic regime. For sake of simplicity, we assume that the ions are cold and consider the three-field model described in a previous paper ${ }^{3}$. Stellarator equilibria have, in general, complicated structure. For example, a typical equilibrium of the three-field-period heliac $\mathrm{H} 1-\mathrm{NF}^{4}$ requires the specification of more than 800 Fourier components for each equilibrium quantity, and for each magnetic surface. Therefore most full-geometry drift wave numerical calculations become prohibitive. In this paper, we study the effect of the magnetic field inhomogeneities (or higher-order equilibrium Fourier components) on the fastest linear growth rate of fluid, electrostatic drift waves. It is shown that not all Fourier components in the representation of the equilibrium configuration are equally important. Although the study has been carried out for one field line, numerical simulations reported here suggest that reduced stellarator equilibria for large-scale (e.g. different field lines and different magnetic surfaces) numerical calculations can be used in specific drift wave problems.

We consider low-frequency, drift-type modes $\left(\omega / \omega_{\text {ci }} \ll 1, k_{\perp} \rho_{\mathrm{i}} \ll 1\right)$ in low- $\beta$, nonaxisymmetric plasmas. The magnetic field is written as $\mathbf{B}=\nabla \alpha \times \nabla \psi$, where $\alpha \equiv \zeta-q \theta$ is the field line label, and $2 \pi \psi$ is the enclosed poloidal flux. For simplicity ions are cold and the parallel velocity of the ions is neglected $\left(v_{\mathrm{ph}}=\omega / k_{\|} \gg v_{\text {thi }}\right)$. The model equations describe the time evolution of the normalized fluctuating potential, density, and electron temperature $\left(\widetilde{\Phi} \equiv e \Phi / T_{\mathrm{e} 0}, \widetilde{n} \equiv \delta n / n_{0}\right.$ and $\widetilde{T}_{\mathrm{e}} \equiv \delta T_{\mathrm{e}} / T_{\mathrm{e} 0}$, respectively). Each fluctuating 
field $\widetilde{F} \equiv \delta F / F_{0} \ll 1$ is written in the eikonal representation as $\widetilde{F}=\widehat{F}(\bar{\zeta}, t) \exp \left(i N_{\mathrm{t}} \alpha\right)$, where $N_{\mathrm{t}} \gg 1$ is the toroidal truncation number, $\widehat{F}$ is a slowly-varying amplitude and $\bar{\zeta}$ is the extended toroidal angle (which labels the position along the field line). Neglecting corrections of order $\mathcal{O}\left[\left(\delta F / F_{0}\right)^{2}\right]$ and higher, the model equations $\operatorname{read}^{3}$

$$
\begin{aligned}
\frac{\partial \widehat{n}}{\partial t} & =i\left(S_{\perp}-S_{\star}\right) \widehat{\Phi} \\
\frac{\partial \widehat{\Phi}}{\partial t} & =\mathcal{L}\left[i S_{\perp}\left(\widehat{n}+\widehat{T}_{\mathrm{e}}\right)-\xi_{\mathrm{c}}\left(L_{n}^{2} \nabla_{\|}^{2} \widehat{F}-L_{n} Q_{\|} \nabla_{\|} \widehat{F}\right)\right], \\
\frac{\partial \widehat{T}_{\mathrm{e}}}{\partial t} & =\xi_{\mathrm{c}}\left(L_{n}^{2} \nabla_{\|}^{2} \widehat{G}-Q_{\|} L_{n} \nabla_{\|} \widehat{G}\right) \\
& +i \widehat{\Phi}\left(\frac{2}{3} S_{\perp}-\eta_{\mathrm{e}} S_{\star}\right)+i \widehat{n}\left(\frac{2}{3} S_{\star}-\frac{2}{3} S_{\perp}\right)+i \widehat{T}_{\mathrm{e}}\left(\frac{2}{3} S_{\star}-\frac{7}{3} S_{\perp}\right),
\end{aligned}
$$

where $t \leftarrow \omega_{\star \mathrm{e}} t$ is the normalized time $\left(\omega_{\star \mathrm{e}}\right.$ is the electron diamagnetic drift frequency), $\widehat{F} \equiv 2\left(1+\mu_{1}\right) \widehat{T_{\mathrm{e}}}+2 \widehat{h}$ where $\widehat{h}=\widehat{n}-\widehat{\Phi}$ is the non-adiabatic response of the electrons $\left(\mu_{1}=0.71\right.$ is a thermoelectric coefficient), $\widehat{G} \equiv 2\left[2\left(1+\mu_{1}\right)^{2}+\mu_{2}\right] \widehat{T_{\mathrm{e}}} / 3+4\left(1+\mu_{1}\right) \widehat{h} / 3, \mu_{2}=3.2$ is a thermoelectric coefficient in the electron parallel heat conductivity, $L_{n} \equiv \bar{a}\left(d n_{0} / d s / n_{0}\right)^{-1}$ is the radial density scalelength ( $a$ is the minor radius of the last closed magnetic surface), $\xi_{\mathrm{c}} \equiv\left(\omega_{\mathrm{ce} 0} \tau_{\mathrm{e}}\right) /\left(k_{\theta} L_{n}\right) \gg 1$ is called the collisional parameter, $\omega_{\mathrm{ce} 0}$ is the electron cyclotron frequency evaluated at the magnetic axis, $\tau_{\mathrm{e}}$ is the electron collision time and $k_{\theta} \equiv N_{\mathrm{t}} q / \bar{a}$. We have also defined $S_{\star} \equiv \bar{a} \sqrt{g^{s s}} \widehat{\mathbf{e}}_{\perp} \cdot\left(\widehat{\mathbf{e}}_{\|} \times \widehat{\mathbf{n}}\right) / B_{\star}$, where $g^{s s}$ is a metric element, $\widehat{\mathbf{n}} \equiv$ $\boldsymbol{\nabla} s /(\boldsymbol{\nabla} s \cdot \boldsymbol{\nabla} s)^{1 / 2}$ is a unit vector normal to the magnetic surface and pointing outwards, $\widehat{\mathbf{e}}_{\|}$ is a unit vector along $\mathbf{B}, \widehat{\mathbf{e}}_{\perp} \equiv \mathbf{k}_{\perp} / k_{\theta} \equiv N_{\mathrm{t}} \boldsymbol{\nabla} \alpha / k_{\theta}$ and $B_{\star}(\bar{\zeta}) \equiv B(\bar{\zeta}) / B_{0}=\mathcal{O}(1)$ is the magnetic field strength normalized to its value at the magnetic axis. As shown in Ref [3], $S_{\star}$ is constant along the magnetic field line. Geometrical effects are contained in the parallel gradient operator, $\nabla_{\|}=\left(B^{-1} \mathbf{B} \cdot \boldsymbol{\nabla}\right)_{\alpha, s}$, the curvature drift term

$$
S_{\perp}(\bar{\zeta}) \equiv \frac{2}{B_{\star}}\left(\widehat{\mathbf{e}}_{\|} \times \widehat{\mathbf{e}}_{\perp}\right) \cdot \mathbf{Q}
$$

where $\mathbf{Q} \equiv L_{n} \boldsymbol{\nabla} B / B$ is related to the magnetic field inhomogeneity; and the polarization drift term $\left(\propto 1 / \mathbf{k}_{\perp} \cdot \mathbf{k}_{\perp}\right)$

$$
\mathcal{L}(\zeta) \equiv \frac{1}{b}\left[\frac{B_{\star}(\zeta)}{\xi_{\perp}(\zeta)}\right]^{2},
$$


where $\xi_{\perp} \equiv\left(\widehat{\mathbf{e}}_{\perp} \cdot \widehat{\mathbf{e}}_{\perp}\right)^{1 / 2}, b=\left(k_{\theta} \rho_{s 0}\right)^{2}, \rho_{s 0}$ is $\rho_{s}=c_{s} / \omega_{\mathrm{ci}}$ evaluated at the magnetic axis. Finally in equation (1), terms proportional to $Q_{\|}=\mathbf{B} \cdot \mathbf{Q} / B$ arise because of the compressibility of the unit vector $\widehat{\mathbf{e}}_{\|}$. Although $Q_{\|}$can be neglected for a large-aspect-ratio tokamak with circular magnetic surfaces, this quantity displays a rapid variation along the field line in the stellarator case (see, e.g, Fig.4 in Ref [3]) and must be retained in the model equations (1). Equations (1) have been solved as an initial-value problem along the field line using a semi-implicit scheme; therefore, the fastest linear growth rate can be obtained without ambiguity. To calculate the stable part of the spectrum, the standard normal mode analysis is more convenient. For each fluctuating quantity $\widetilde{F}$, a dynamical growth rate can calculated as $\gamma_{F}(t)=\langle|\widehat{F}|\rangle^{-1} \partial\langle|\widehat{F}|\rangle / \partial t$ where $|\widehat{F}|=\left(\widehat{F} \widehat{F}^{\star}\right)^{1 / 2}$ and, for any $G(\zeta),\langle G\rangle$ denotes an average over the extended toroidal angle

$$
\langle G\rangle \equiv \frac{1}{2 \zeta_{\mathrm{m}}} \int_{\zeta_{0}-\zeta_{\mathrm{m}}}^{\zeta_{0}+\zeta_{\mathrm{m}}} G\left(\zeta^{\prime}\right) d \zeta^{\prime}
$$

where $\zeta_{0}$ is a toroidal angle of reference and $\zeta_{\mathrm{m}}$ is an adjustable parameter. As shown in Ref [3], $\gamma_{n} / \gamma_{\Phi} \mapsto 1$ and $\gamma_{T_{\mathrm{e}}} / \gamma_{\Phi} \mapsto 1$ as $t \mapsto \infty$. For $t \gg 1$, we denote the final growth rate as $\gamma$. The geometrical terms must be calculated in curvilinear coordinates, which involves metric elements of the form $g^{\mu \lambda}=\nabla \mu \cdot \nabla \lambda$, for $(\mu, \lambda)=\{s, \theta, \zeta\}$. The equilibrium has been computed for the three-field-period toroidal heliac $\mathrm{H} 1-\mathrm{NF}^{4}$ using the VMEC code ${ }^{5,6}$ with fixed boundary conditions, zero net toroidal current and a volume-averaged $\beta$ of $0.36 \%$ for a set of 100 magnetic surfaces; the relevant equilibrium quantities are then mapped from VMEC coordinates to Boozer coordinates. The position vector on a given magnetic surface, $s=$ const, can be written in cylindrical coordinates $\mathbf{r}=R \cos \phi \widehat{\mathbf{x}}+R \sin \phi \widehat{\mathbf{y}}+Z \widehat{\mathbf{z}}$ where

$$
\begin{aligned}
R & =\sum_{m=0}^{M_{\max }} \sum_{n=-N_{\max }}^{+N_{\max }} R_{m n} \cos \left(\mu_{m n}\right) \\
Z & =\sum_{m=0}^{M_{\max }} \sum_{n=-N_{\max }}^{+N_{\max }} Z_{m n} \sin \left(\mu_{m n}\right) \\
\phi & =\zeta-\frac{2 \pi}{N_{\mathrm{p}}} \sum_{m=0}^{M_{\max }} \sum_{n=-N_{\max }}^{+N_{\max }} \widetilde{\phi}_{m n} \sin \left(\mu_{m n}\right)
\end{aligned}
$$

where $\mu_{m n} \equiv m \theta+N_{\mathrm{p}} n \zeta, N_{\mathrm{p}}=3$ is the number of field periods and $M_{\max }=13, N_{\max }=27$. 
Therefore, the calculation of the cylindrical radius $R$ alone requires the calculation of $N_{\text {tot }}$ cosine terms, where $N_{\text {tot }}=\left(M_{\max }+1\right)\left(2 N_{\max }+1\right)=812$ is the number of equilibrium Fourier components (for one magnetic surface). Numerical calculations can be become rapidly prohibitive. Consider, for example, the contravariant basis vector $\nabla s$, which can be written as $\boldsymbol{\nabla} s=\mathcal{J}^{-1} \mathbf{e}_{\theta} \times \mathbf{e}_{\zeta}$, where $\mathbf{e}_{\theta}=\partial \mathbf{r} / \partial \theta$ and $\mathbf{e}_{\zeta}=\partial \mathbf{r} / \partial \zeta$ are covariant basis vectors, and $\mathcal{J}=[\boldsymbol{\nabla} s \cdot(\boldsymbol{\nabla} \theta \times \boldsymbol{\nabla} \zeta)]^{-1}=\mathbf{e}_{s} \cdot\left(\mathbf{e}_{\theta} \times \mathbf{e}_{\zeta}\right)$ is the Jacobian of the transformation. To calculate $\mathbf{e}_{\theta}$ and $\mathbf{e}_{\zeta}$ we use the expression for the position vector $\mathbf{r}$ in cylindrical coordinates; this yields $\mathbf{e}_{\mu}=[\cos \phi(\partial R / \partial \mu)-R \sin \phi(\partial \phi / \partial \mu)] \widehat{\mathbf{x}}+[\sin \phi(\partial R / \partial \mu)+R \cos \phi(\partial \phi / \partial \mu)] \widehat{\mathbf{y}}+\partial Z / \partial \mu \widehat{\mathbf{z}}$, for $\mu=\{s, \theta, \zeta\}$. Thus the numerical calculation of $\nabla s$ along the field line involves the evaluation of $3 \times 3 \times N_{\text {tot }} \sim 7 \times 10^{4}$ sine or cosine terms. To reduce the CPU time on computing the geometrical terms in the model equations (1), one possible approach consists in Fourier transforming the curvature term $S_{\perp}$

$$
S_{\perp}(\bar{\zeta})=S_{\perp}^{[\mathrm{S}]}(\bar{\zeta})+\sum_{p} S_{\perp p} \cos (p \bar{\zeta})
$$

where, as before, $\bar{\zeta}$ is the extended toroidal angle, and $S_{\perp}^{[\mathrm{S}]}$ is the secular part of $S_{\perp}$. We have assumed that the reference $\bar{\zeta}=0$ corresponds to a symmetry point, so that for any equilibrium quantity $G$, one has $G(+\bar{\zeta})=G(-\bar{\zeta})$. However the presence of a secular part in $S_{\perp}$ requires the storage of $S_{\perp}^{[\mathrm{S}]}$ at each grid point.

Alternatively, we can truncate the Fourier series (5) with $M \leq M_{\max }$ and $N \leq N_{\max }$. As a consequence the linear growth rate will depend on how many Fourier components are retained; so we write $\gamma=\gamma(M, N)$, the 'exact' (full equilibrium) growth rate being $\gamma_{0} \equiv \gamma\left(M_{\max }, N_{\max }\right)$. As in Ref [3], we choose the field line passing at the outside of the torus, with $\theta_{0}=\zeta_{0}=0$. The magnetic surface is $s_{0}=0.9$, where the global magnetic shear is slightly negative. Other parameters are $k_{\theta} \rho_{s 0}=0.8, T_{\mathrm{e} 0}=25 \mathrm{eV}, L_{n}=3.0 \mathrm{~cm}, \eta_{\mathrm{e}}=1.5$, $n_{0}=5 \times 10^{12} \mathrm{~cm}^{-3}$. The (normalized) time step is $\Delta t=10^{-3}$ (an explicit scheme would require $\Delta t<10^{-5}$ ). The growth rate calculated with the full equilibrium is $\gamma_{0}=1.323$. Figure 1 shows the linear growth $\gamma$ as a function of the equilibrium poloidal truncation 
number $M$ (with $N=N_{\max }$ ) for the cases $\eta_{\mathrm{e}}=1.5$ (squares) and $\eta_{\mathrm{e}}=0$ (diamonds). As can be expected $\gamma$ reaches $\gamma_{0}$ as $M \mapsto M_{\text {max }}$. However, in the case $\eta_{\mathrm{e}}=1.5$, the convergence is slow. Figure 2 shows $\gamma$ as a function of the equilibrium toroidal truncation number $N$ (with $M=M_{\max }$ ) for the cases $\eta_{\mathrm{e}}=1.5$ (squares) and $\eta_{\mathrm{e}}=0$ (diamonds). Interestingly, in both cases, the linear growth rate reaches its asymptotic value for small values of $N$. For instance, the calculations with $M=M_{\max }$ and $N=6$ yield approximately the correct growth rate $\gamma_{0}$. In this case, the number of elements in each of the matrices $R_{M \times N}, Z_{M \times N}$ and $\widetilde{\phi}_{M \times N}$ is $N_{\text {tot }}=182$ which roughly corresponds to $1 / 5$ of the full equilibrium. We note that the small $\eta_{\mathrm{e}}$ case is far less sensitive than the large $\eta_{\mathrm{e}}$ case. The variation of $\gamma(M, N)$ in the $M-N$ plane (for $\eta_{\mathrm{e}}=1.5$ ) is shown in Fig. 3. Clearly not all Fourier components in specifying the equilibrium are equally important. Although the dependence of $\gamma(M, N)$ should be mode dependent, the geometrical terms, such as the curvature term $S_{\perp}(\bar{\zeta})$ and the polarization term $\mathcal{L}(\bar{\zeta})$, enter the model equations in a similar way, at least in the linear formalism. For a particular fluid model and for a specific field line, it has been shown that the fastest linear growth rate $\gamma$ is rather insensitive to the equilibrium toroidal truncation number $N$, but varies (slowly) with the equilibrium poloidal truncation number $M$. It is possible to compare the magnitude of the Fourier coefficients of the curvature term, $S_{\perp}(\bar{\zeta})$, and the polarization term, $\mathcal{L}(\bar{\zeta})$; however, this term-by-term comparison cannot be done for the $\nabla_{\|}$and $\nabla_{\|}^{2}$ operators. Consider, for instance, the parallel gradient operator

$$
\nabla_{\|}=\frac{S_{\|}(\bar{\zeta})}{\bar{R}} \frac{\partial}{\partial \bar{\zeta}}
$$

For a tokamak with concentric, circular magnetic surfaces, we have $\bar{\zeta} \mapsto q \bar{\theta}, \bar{R} \mapsto R_{0}$, $S_{\|}(\bar{\theta})=1$, so that the usual expression $\nabla_{\|}=\left(q R_{0}\right)^{-1} \partial / \partial \bar{\theta}$ is recovered. In a stellarator, the modulation $S_{\|}$along the field line cannot be neglected. Therefore, this yields a 'scrambling' of equilibrium Fourier components in the parallel direction.

Although our model uses fluid equations, a reduced equilibrium can perhaps also be useful for gyro-kinetic particle codes. 


\section{ACKNOWLEDGMENTS}

The author was supported by a Natural Sciences and Engineering Research Council of Canada (NSERC) Research Fellowship. 


\section{REFERENCES}

${ }^{1}$ W. M. Tang, Nucl. Fusion 18, 1089 (1978).

${ }^{2}$ S.I. Braginskii, in Review of Plasma Physics, edited by M.A. Leontovitch (Consultants Bureau, N.Y., 1965).

${ }^{3}$ J.L.V. Lewandowski, Phys. Plasmas 4(11), 4023 (1997).

${ }^{4}$ S.M. Hamberger, B.D. Blackwell, L.E. Sharp, and D.B. Shenton, Fus. Tech. 17, 123 (1990).

${ }^{5}$ S.P. Hirshman and J.C. Whitson, Phys. Fluids 26, 3553 (1983).

${ }^{6}$ S.P. Hirshman and H.K. Meier, Phys. Fluids 28, 1387 (1985). 
Fig.1 Fastest linear growth rate $\gamma$ as a function of the equilibrium Fourier poloidal truncation number $M$ for $\eta_{\mathrm{e}}=1.5$ (squares) and $\eta_{\mathrm{e}}=0$ (diamonds). The equilibrium Fourier toroidal truncation number is $N=N_{\max }=27$.

Fig.2 Fastest linear growth rate $\gamma$ as a function of the equilibrium Fourier toroidal truncation number $N$ for $\eta_{\mathrm{e}}=1.5$ (squares) and $\eta_{\mathrm{e}}=0$ (diamonds). The equilibrium Fourier poloidal truncation number is $M=M_{\max }=13$.

Fig.3 Fastest linear growth rate in the $M-N$ plane for the case $\eta_{\mathrm{e}}=1.5$. 
FIG.1 Lewandowski

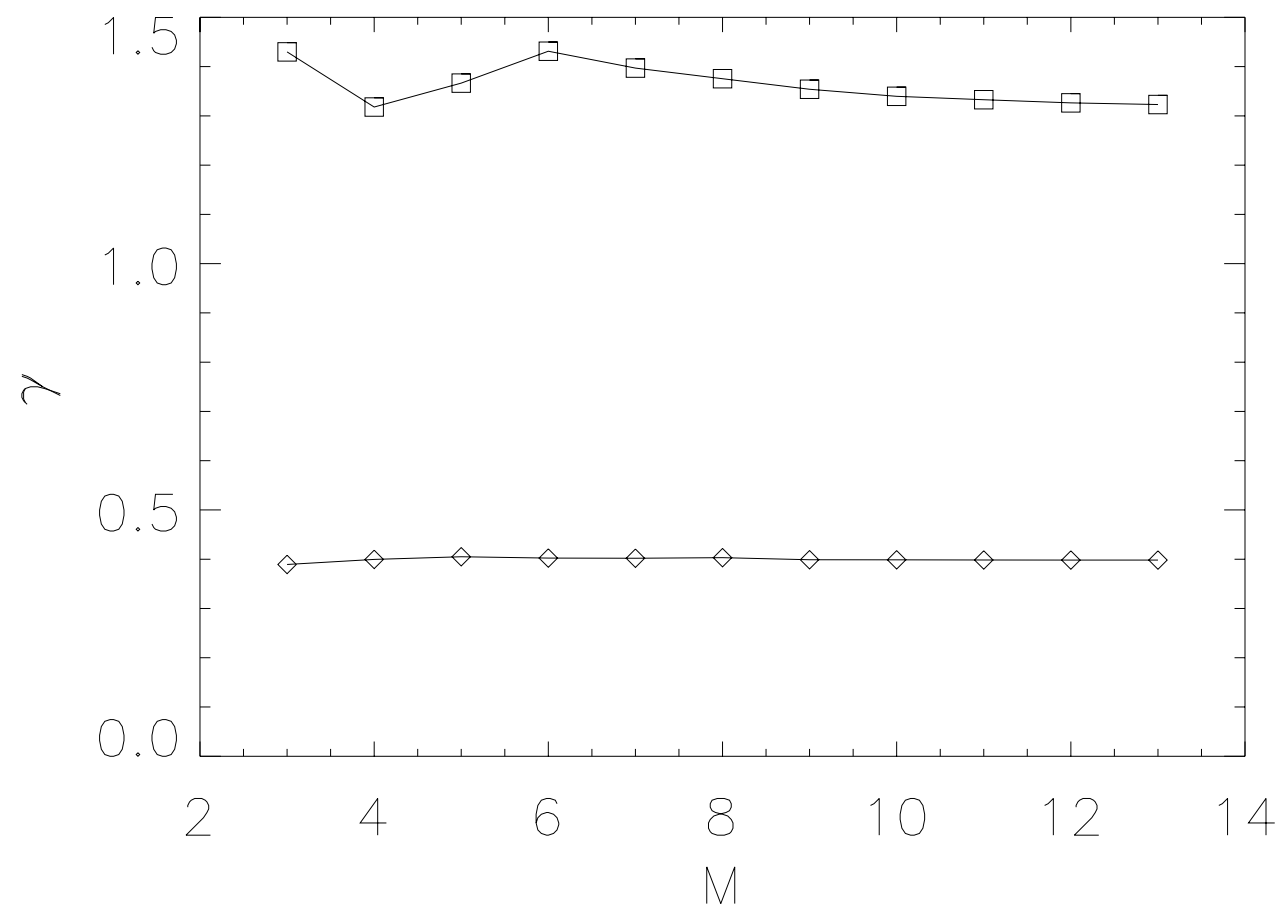


FIG.2 Lewandowski

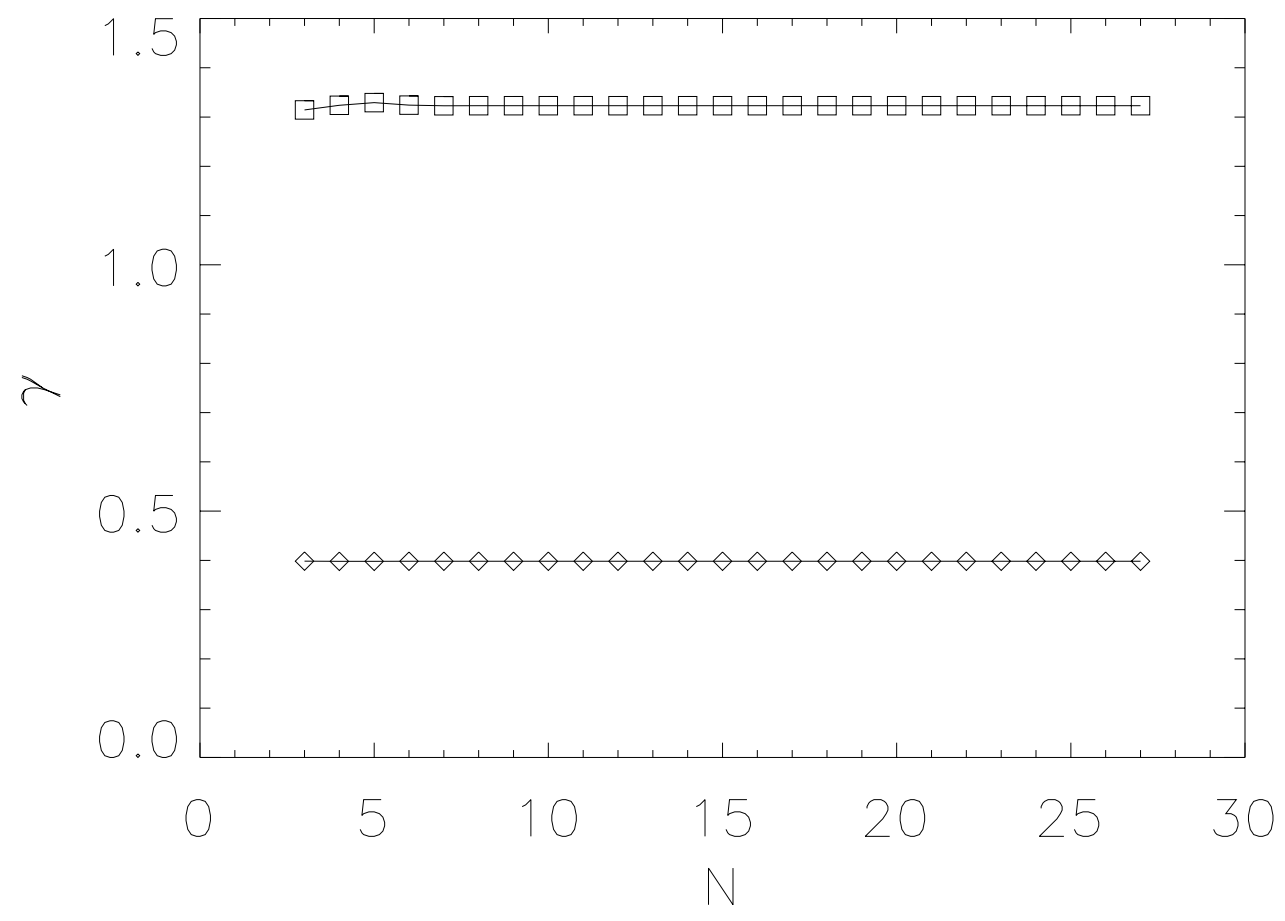


FIG.3 Lewandowski

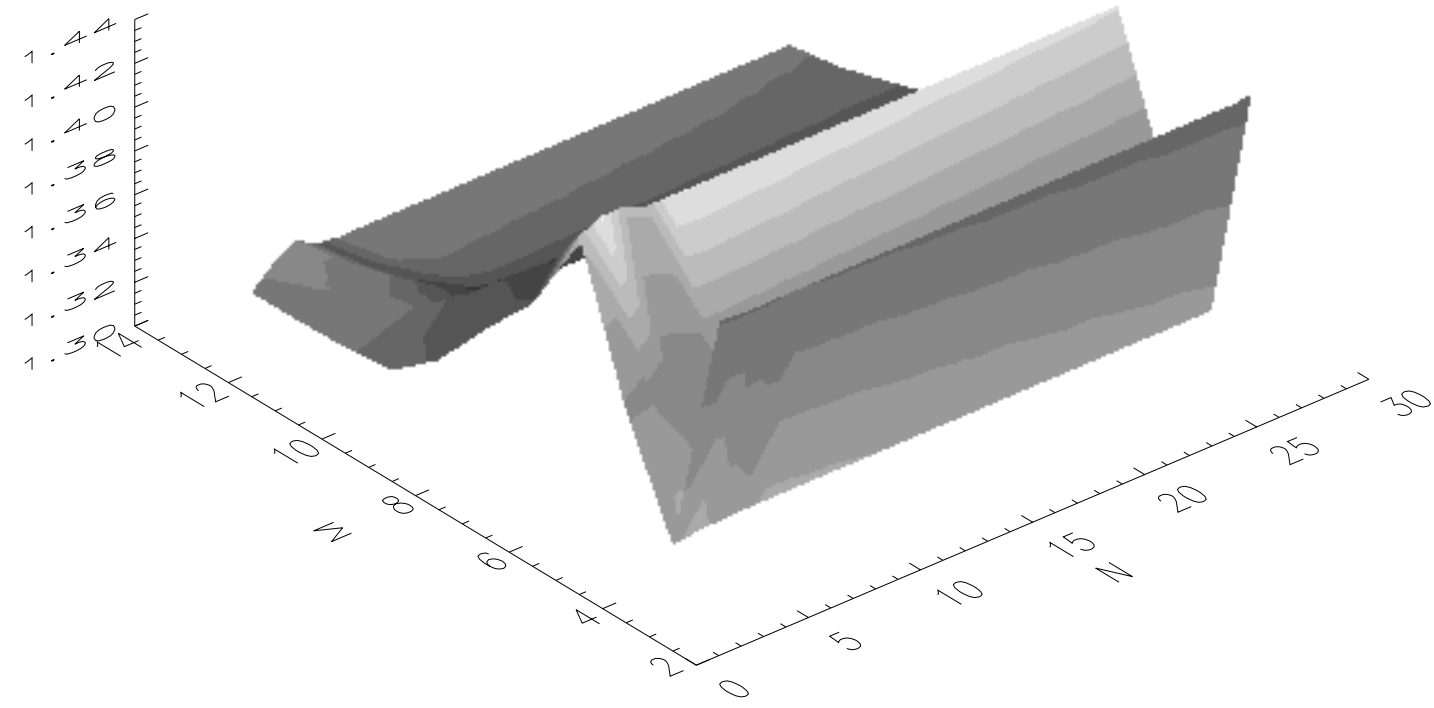

